\title{
Surface-Enhanced Raman Spectroscopy using silver impregnated polycarbonate substrates
}

\author{
L. Lagonigro, A. C. Peacock, P. J. A. Sazio \\ Optoelectronics Research Centre, University of Southampton, SO17 1BJ, UK \\ T. Hasell, P. D. Brown and S. M. Howdle \\ School of Chemistry, University of Nottingham, NG7 2RD, UK
}

Novel substrates based on noble metal nanoparticles are currently the subject of extensive research in fields such as biological sensing, medicine, spectroscopy and nano-photonics due to the large electromagnetic fields generated in the vicinity of the metal surface via a surface plasmon resonance. The dependence of the resonance wavelength on the size, shape, local dielectric environment and interparticle spacing, enables engineering of the metal nanoparticle substrates to target specific requirements [1]. Here we report the fabrication of silver impregnated polycarbonate composites as substrates for surface enhanced Raman spectroscopy (SERS) where the plasmonic properties can be controlled via the reaction parameters. Although silver has superior plasmonic properties to other metals, it is often over looked for SERS substrates as it is highly prone to oxidation. The embedding of silver nanoparticles into polymer substrates offers substantial environmental protection, allowing for the construction of temporally stable plasmonic devices that can exploit the mechanical flexibility of the polymers and prevent particle agglomeration. These nanoparticle composites offer a number of advantages as SERS substrates as they are cheap, easily processed, and are bio-compatible.

Fabrication of the substrate is based on supercritical carbon dioxide processing to produce silver nanoparticles in situ within prefabricated optically transparent polycarbonate strips. The substrates have been characterised by TEM, UV-Vis absorption and Raman scattering measurements. Fig. 1(a) shows a TEM micrograph of a cross section of the silver-polycarbonate strip revealing a nanoparticle band of uniform thickness $(\sim 5.5 \mu \mathrm{m})$ and composition along the length of the sample. The inset shows a close up of the SERS active nanoparticles near the surface of the film, where we can estimate the size of the nanoparticles to be $\sim 15 \mathrm{~nm}$. UV-Vis absorption measurements plotted in Fig. 1(b) show a surface plasmon resonance around $410 \mathrm{~nm}$, in agreement with calculations based on Mie scattering. The intensity of the peak increases with the amount of precursor used, indicating an increase in the concentration of nanoparticles produced.

To test the silver-polycarbonate substrates for SERS activity, we drop cast $0.5 \mathrm{mM}$ of aminothiophenol in ethanol onto the silver surface. The Raman spectrum for a sample with a high concentration of nanoparticles at the surface is plotted in Fig. 1(c) (black line) clearly showing the main vibrational modes of aminothiophenol as assigned to those reported in the literature [2]. Additionally no Raman peaks of the target molecule were observed on a control undoped polycarbonate sample treated in the same manner (grey line). SERS spectra collected a month after the aminothiophenol deposition produced results of comparable intensity, confirming the temporal stability of the substrates. We will discuss alternative excitation geometries via waveguiding in the high index metal nanoparticle strip using simple lithographic processing techniques.
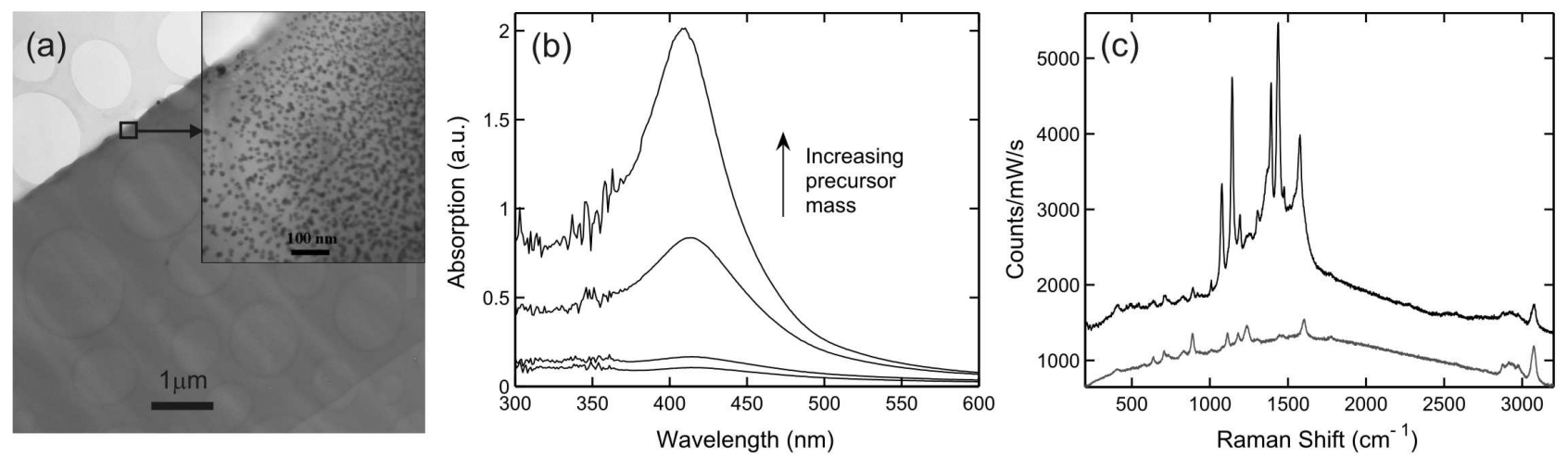

Fig. 1 a) TEM imaging of silver-polycarbonate cross-section. The inset shows a close up of the SERS active nanoparticles near the surface. b) UV-Vis spectra for various precursor concentrations. c) Raman spectra of aminothiophenol deposited on silver-polycarbonate strip (black line) and on undoped polycarbonate sample (grey line).

\section{References}

[1] E. Hutter and J. H. Fendler "Exploitation of Localized Surface Plasmon Resonance," Adv. Mater. 16, 1685 (2004)

[2] M. Osawa et al. "Charge Transfer Resonance Process in Surface-Enhanced Raman Scattering from $p$ -

Aminothiophenol Adsorbed on Silver: Herzberg-Teller Contribution,” J. Phys. Chem. 98, 12702 (1994) 